\title{
Lyapunov Observer/Controller for Stable Haptic Interaction
}

\author{
Aghil Jafari, Member, IEEE, Harsimran Singh, Member, IEEE, Harsha Karunanayaka, \\ Jee-Hwan Ryu, Member, IEEE, Jj Chong and Appolinaire C. Etoundi
}

\begin{abstract}
Passivity has been the most common tool to achieve stability in haptic and teleoperation systems; however passivity-based approaches suffer from the conservativism of passivity criteria. Therefore, it is essential to have an approach which is less conservative than conventional approaches, enabling the user to have an immersive experience when interacting with the virtual or remote environment. This paper proposes a Lyapunov observed and controller (LOLC) which has an improved transparency as it uses the Lyapunov boundedness theorem that is less conservative than passivity theorem. The proposed approach also gives the user the freedom to define a relaxed Lyapunov candidate regarding state stability to be chosen. Moreover, the application of the proposed control architecture is not limited to haptic interfaces, and the proposed control methodology could be used to stabilize any control systems. The advantages of the proposed approach to the passivity observer and controller are highlighted via numerical evaluations.
\end{abstract}

\section{INTRODUCTION}

Significant number of researches have been done on achieving stable haptic interaction. Most of these researches are based on the passivity criterion [1], [2]. It is an energybased method where it defines a system is passive if and only if the energy flowing in exceeds the energy flowing out of the system for all time. It uses only the input/output information independent of system parameters. Moreover, passivity criterion can be used in linear and non-linear systems, and it is a sufficient condition for system stability. With the influence of passivity criterion, several approaches have been proposed for stable haptic interaction. The time domain passivity approach (TDPA) proposed by Hannaford and Ryu [2] introduces an adaptive virtual damping to satisfy the passivity constraints. The energy-bound algorithm which was proposed by Kim and Ryu [3], blocks the generated energy of a ZOH in a way that haptic system does not possess excessive energy. Ryu and Yoon [4] proposed a memorybased passivation approach to increase the dynamic range of an impedance that a multiple-degree-of-freedom (multi DoF)

Aghil Jafari, Appolinaire C. Etoundi, and $\mathrm{Jj}$ Chong are with the Faculty of Environment and Technology, University of the West of England, Bristol, United Kingdom. Email: \{aghil.jafari, appolinaire.etoundi,jj.chong\}@uw e.ac.uk

Harsimran Singh is with the Institute of Robotics and Mechatronics in the German Aerospace Center (DLR), 82234 Wessling, Germany. Email: harsimran.singhedlr.de

Harsha Karunanayaka is with the Faculty of Engineering, University of Bristol, Bristol, United Kingdom. Email: hk17441@my.bristol.ac.uk

Jee-Hwan Ryu is with the School of Mechanical Engineering, Korea University of Technology and Education (KoreaTech), Rep. of Korea. Email: jhryuekoreatech.ac.kr haptic interface can passively render. Even though these approaches can stabilize the haptic interaction with high stiffness Virtual Environments (VEs), the passivity constraint which is known to be conservative, limits the performance each these approaches due to having a small subset of the entire stability region. Therefore, the actual apparent impedance is smaller than the desired impedance in a notable way.

To address the conservatism of the passivity-based controllers, several approaches have been proposed based on the absolute stability and the input-to-state stable (ISS) criteria. A two-port network is known to be absolute stable, if a set of passive terminating one-port impedances for which the system is unstable does not exist. Therefore, if the haptic interface is designed to be absolute stable, the haptic simulation continues to be stable unless the human operator and VE are active. For the absolute stability of linear twoport networks, the Llewellyns stability criterion provides both necessary and sufficient conditions [5]. However, the sufficient conditions of the absolute stability are represented in frequency domain which are unable to determine in many real-time systems. Jafari and et al. [6] proposed the ISS approach to address this issues of absolute stability criterion in haptic interfaces. A system is known to be ISS if and only if it is dissipative [7], [8]. Therefore, by using a control framework including a gain which is the max slope of the input-output graph, the sufficient condition required for the dissipativity is satisfied, then the system becomes input-tostate stable [9]. This approach makes the output state of the system bounded. However, for some applications this bounded behaviour may be considered as unstable behaviour for the operator. To address this issue an extra feed-forward gain is introduced to the controller. Yet the value of the gain of this feed forward pass is limited by noise and the application of the system.

To overcome the aforementioned issues, this paper comes with the idea of Lyapunov Observer and Lyapunov Controller (LOLC). First, for the observer part, the operator must propose a Lyapunov candidate, and for the controller part, the satisfaction of Lyapunov boundedness theorem should be provided. The contribution of this work not only lies on the fact the Lyapunov boundedness theorem is less conservative than passivity theorem, but also it gives the operator the ability to choose whatever relaxed Lyapunov candidate in the terms of state' stability to be chosen. As the result, the overall controller framework possibly could be less conservative compared with the energy-based approaches where they have a fixed criterion to satisfy. Note that the proposed LOLC is 
different from the conventional adaptive controllers where the adaptive law is derived from a Lyapunov candidate. Unlike the conventional adaptive controllers, the proposed LOLC does not use the dynamics model of the system and it does not update the system parameters but the LC. In addition, in this paper, the LOLC has been proposed and demonstrated for haptic interfaces, the main idea of LOLC is not limited to haptic interfaces, and it could be applied to any control systems. Numerical analysis has been provided to show the effectiveness and feasibility of the proposed approach.

The rest of the paper is organized as follows. section II describes the concept of Lyapunov boundedness theorem and the sufficient conditions for a system to be output bounded in terms of Lyapunov stability. Next, section III proposes the Lyapunov observer and controller for stable haptic interaction with a VE. Then, section IV provides the numerical evaluation. Finally, Section V concludes the paper.

\section{LyAPUNOV FUNCTION AND BOUNDEDNESS}

Lyapunov second method for the stability introduce the Lyapunov function $V(x)$ which has an analogy to the potential function of classical dynamics. The following theorem explains the necessary conditions for a Lyapunov function candidate for any system.

Theorem 2.1: [10] Let $x_{e}=0$ be and equilibrium point for the autonomous system $\dot{x}=f(x)$ and $D \subset \mathbb{R}^{n}$ be a domain containing $x=0$. Let a continuously differentiable function $V: D \rightarrow \mathbb{R}$ such that,

$$
\begin{gathered}
V(0)=0 \text { and } V(x)>0 \text { in } D-\{0\} \\
\dot{V}(x) \leq 0 \text { in } D
\end{gathered}
$$

Then $x=0$ is known to be stable. Also if;

$$
\dot{V}(x)<0 \quad \text { in } \quad D-\{0\}
$$

Then $x=0$ is known to be symptomatically stable.

A continuously differentiable function $V(x)$ that satisfy eq. (1) and eq. (2) is known as a Lyapunov function (LF).

Fig. 1 shows the level surface of a lyapunov function $V(x)$ where the $V(x)=c$, for some $c>0$. The rate of change of the function $V(x) ; \dot{V}(x) \leq 0$ implies that when a trajectory crosses the Lyapunov Surface (LS) $V(x)=c$, it moves inside the set $\Omega_{c}$ such that; $\Omega_{c}=\left\{x \in \mathbb{R}^{n} \mid V(x) \leq c\right\}$ and never come out again. When $\dot{V}(x)<0$, the trajectory moves from one LS to and inner LS with a smaller $c$ value and as the value of $c$ decreases, the $\operatorname{LS} V(x)=c$ shrink to the origin. This phenomenon shows that, the trajectory approach toward the origin when $t \rightarrow \infty$.

Therefore, we can redefine the Lyapunov's theorem with this terminology as; if there is a continuously differentiable positive definite function $V(x)$ so that $\dot{V}(x)$ is negative semidefinite, the origin said to be stable whereas, if $\dot{V}(x)$ is negative definite, origin is said to be asymptotically stable.

This Lyapunov approach can be used to show boundedness of the solution of a state equation, even when there is no equilibrium point at the origin. To testify this phenomenon;

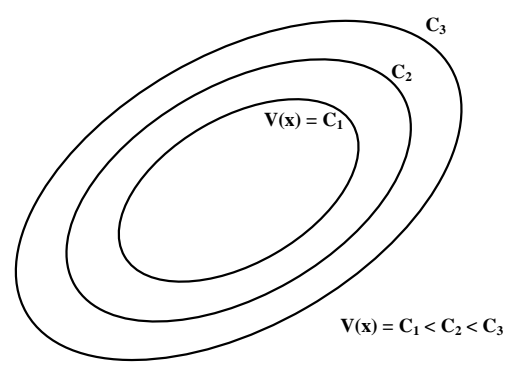

Fig. 1: Level surface of a Lyapunov function [10]

Suppose there is a positive definite Lyapunov candidate $V(x): \mathbb{R}^{n} \rightarrow \mathbb{R}$ that satisfies,

- All sub-level sets of $V$ are bounded

- $\dot{V}(x) \leq 0$ for all $x$

then, all trajectories are bounded. For each trajectory $x$ there is an $R$ such that $\|x(t)\| \leq R$ for all $t \geq 0$. Therefore, it can be defined that all the trajectories (or outputs) are bounded if;

$$
V(x(t))=V(x(0))+\int_{0}^{t} \dot{V}(x(\tau)) d \tau \leq V(x(0))
$$

Integral term in eq. (4) shows how the trajectory of the system bounded between $V(x(0))$ and $V(x(t))$, where, $V(0)$ is the origin of the function. When the value of the integral term increases with the time and since that value is minus (according to the condition $\dot{V}(x) \leq 0), V(x(t)) \rightarrow V(0)$. Furthermore, using eq. (1), eq. (4) can be reduce to,

$$
\int_{0}^{t} \dot{V}(x(\tau)) d \tau \leq 0
$$

The above shows the condition that need to be be satisfied by a continuous-time system. Therefore, for a discrete-time system eq. (5) can be rewritten as;

$$
\triangle t \sum_{k=0}^{n} \dot{V}(x(k)) \leq 0
$$

where $n$ is the sampling time of the discrete-time system, and $\Delta t$ is the sampling time step. Because $\Delta t$ is positive, the above equation could be reformulated as:

$$
\sum_{k=0}^{n} \dot{V}(x(k)) \leq 0
$$

\section{LYAPUNOV OBSERVER AND CONTROLLER FOR STABLE HAPTIC INTERACTION}

In this section, the Lyapunov Observer (LO) and Lyapunov Controller (LC) are proposed for a one-port network system as shown in the Fig. 2. Then, in the following, the idea is explained via two examples.

The control architecture of the proposed Lyapunov observer/controller is based on the Lyapunov boundedness 


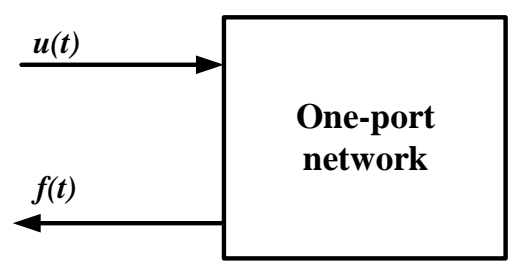

Fig. 2: One-port network system

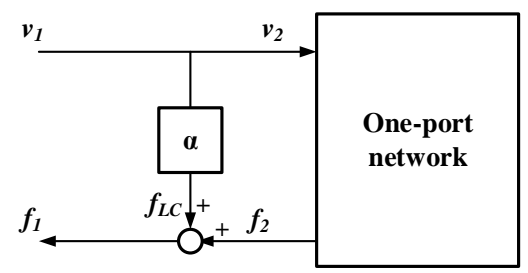

Fig. 3: Lyapunov controller for one-port system

theorem (eq. (7)) which is less conservative than the passivity theorem by allowing the system to generate energy as long as the output is bounded. Moreover, unlike the passivity theorem, it gives the operator the opportunity to choose whatever required relaxed Lyapunov candidate to be chosen. Note that, the proposed is not limited for haptic interaction and if we are able to introduce a Lyapunov candidate for any closed loop of the system, it is possible that to extend the proposed approach for any control architecture.

The Lyapunov candidate must be a positive definite function (eq. (1)), and the selection of the function $V(z)$ is arbitrary. Then, the terminology of LO is based on the satisfaction of eq. (7). Thus, we can implement the LO as the following to see whether it satisfy the eq. (7) or not:

$$
L_{o b s v}(n)=\sum_{k=0}^{n} \dot{V}(x(k))
$$

By considering the reaction force $(-f)$ of the $\mathrm{VE}$ which is applied to the operator, we can say that if $L_{\text {obsv }}(n) \geq 0$ for every $n$, then the output of the system is bounded. Otherwise, if $L_{o b s v}(n)<0$ at any instance, then possibly the system's output is not bounded, and the system is deviated from the Lyapunov criterion by $-L_{o b s v}(n)$. Then, the LC is used to compensate the deviation $\left(-L_{o b s v}(n)\right)$ through an adjustable damping element $\alpha$ as shown in Fig.5. Because, we know the exact amount of the deviation $\left(-L_{o b s v}(n)\right)$, by adjusting the value of $\alpha$, the energy could be dissipated leading the system to be stable based on Lyapunov boundedness theorem (eq. (7)) . Fig. 3 shows the LC for impedance causality of a one-port network. In the following, the formulation of $\mathrm{LO} / \mathrm{LC}$ is explained through two examples.

\section{A. Example 1}

Let's consider a virtual mass-spring system with a mass $m$ and a stiffness $k$. To make the system more simple, the

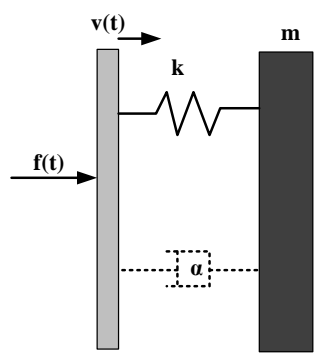

Fig. 4: Virtual environment with a mass and a spring

mass of the system $m$ is neglected. Fig.4 show the schematic diagram of the simple system. The $\alpha$ value for the LC, for the impedance causality of the system can be calculated as follows.

The force that is acting on the system could be described as:

$$
-f=k x+\alpha \dot{x}
$$

The Lyapunov candidate is selected by considering the total energy of the system. It can be written as,

$$
V=\frac{k x^{2}}{2}
$$

Then, the time derivation is;

$$
\dot{V}=k x \dot{x}=-f \dot{x}-\alpha \dot{x}^{2}
$$

Then, the value of $\alpha$ for the above system could be calculated as follows:

$$
\begin{gathered}
\text { if } \sum_{k=0}^{n} \dot{V}>0, \text { and then to make the system stable; } \\
\sum_{k=0}^{n}-f(k) \dot{x}(k)+\sum_{k=0}^{n}-\alpha(k) \dot{x}^{2}(k)=0 \\
\sum_{k=0}^{n}-f(k) \dot{x}(k)+\sum_{k=0}^{n-1}-\alpha(k) \dot{x}^{2}(k)-\alpha(n) \dot{x}^{2}(n)=0
\end{gathered}
$$

Then the $\alpha(n)$ can be calculated as;

$$
\alpha(n)=\frac{-\sum_{k=0}^{n} f(k) \dot{x}(k)-\sum_{k=0}^{n-1} \alpha(k) \dot{x}^{2}(k)}{\dot{x}^{2}(n)}
$$

Using eq. (8), eq. (11) and eq. (12);

$$
\alpha(n)=\frac{-L_{o b s v}}{\dot{x}^{2}(n)}
$$

The above equation shows the $\alpha$ value to make the system stable if it is not stable by itself based on the Lyapunov boundedness theorem. It is the same result that Hannaford and Ryu [2] obtained using the POPC approach. Hence, for a virtual spring system, the LC could be implemented as follows:

1) Input is such that; $v_{1}(n)=v_{2}(n)$.

2) $f_{2}(n)=F_{V E}\left(v_{2}(n)\right)$, where $F_{V E}$ is the output force of the VE. 


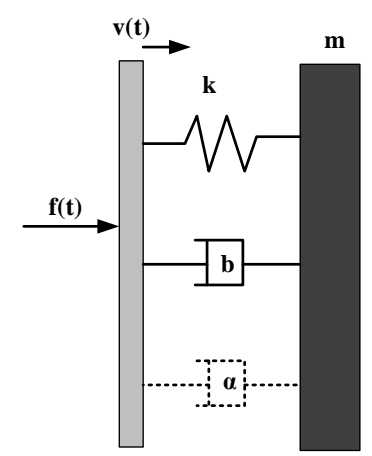

Fig. 5: Virtual environment with a mass, spring and damper

3) Selection of the Lyapunov candidate for the LO (eq. (8))

4) $L_{o b s v}(n)=L_{o b s v}(n-1)+\left(f_{2}(n) v_{2}(n)+\alpha(n-\right.$ 5) 1) $\left.v_{2}(n-1)^{2}\right)$

$$
\alpha(n)= \begin{cases}-L_{o b s v} / v_{2}(n)^{2} & \text { if } L_{o b s v}<0 \\ 0 & \text { if } L_{o b s v} \geq 0\end{cases}
$$

6) Output $\Longrightarrow f_{1}(n)=f_{2}(n)+\alpha(n) v_{2}(n)$

In the next example, the proposed LOLC approach is introduced to a mass-spring-damper system to make it stable.

\section{B. Example 2}

Let's consider a VE (Fig. 5) with a mass $(m)$, spring $(k)$ and damper $(b)$. The input for the system is velocity $(v(t))$ and the output is force $(f(t))$.

By equilibrium of the forces that acting on the system;

$$
-f=m \ddot{x}+(b+\alpha) \dot{x}+k x
$$

The Lyapunov candidate is selected as,

$$
V=\frac{k x^{2}}{2}+\frac{m \dot{x}^{2}}{2}
$$

Then, the $\dot{V}$ is;

$$
\dot{V}=k x \dot{x}+m \dot{x} \ddot{x}=-f \dot{x}-b \dot{x}^{2}-\alpha \dot{x}^{2}
$$

Using the eq. (16), $\alpha$ can be calculated as follows. if $\sum_{k=0}^{n} \dot{V}>0$, and then to make the system stable;

$$
-\sum_{k=0}^{n} f(k) \dot{x}(k)-\sum_{k=0}^{n} \alpha(k) \dot{x}(k)^{2}-\sum_{k=0}^{n} b \dot{x}(k)^{2}=0
$$

The above equation can be rearranged as;

$$
\begin{aligned}
\dot{V}(n) & =-\sum_{k=0}^{n} f(k) \dot{x}(k)-\sum_{k=0}^{n} b \dot{x}(k)^{2} \\
& -\sum_{k=0}^{n-1} \alpha(k) \dot{x}(k)^{2}-\alpha(n) \dot{x}(n)^{2}=0
\end{aligned}
$$

Then, the $\alpha(n)$ can be calculated as;

$$
\alpha(n)=\frac{-\sum_{k=0}^{n} f(k) \dot{x}(k)-\sum_{k=0}^{n} b \dot{x}(k)^{2}-\sum_{k=0}^{n-1} \alpha(k) \dot{x}(k)^{2}}{\dot{x}(n)^{2}}
$$

Using the eq. (8), eq. (16), and eq. (17);

$$
\alpha(n)=\frac{-L_{o b s v}}{\dot{x}(n)^{2}}
$$

By considering the disspated energy by the damper (b) to be monitored without detecting it as an active energy, the above equation is the less conservative version of the PO. The calculation of $\alpha$ for both simple and complex systems is similar (eq. (13), eq. (18)). However, $L_{o b s v}$ in example 2 , is the less relaxed version of $L_{o b s v}$ in example 1 . Note that, LC with impedance causility for this example can be implemented as follows.

1) Input is such that; $v_{1}(n)=v_{2}(n)$.

2) $f_{2}(n)=F_{V E}\left(v_{2}(n)\right)$, where $F_{V E}$ is the output force of the VE.

3) Selection of the Lyapunov candidate for the LO (eq. (8)).

4) $L_{o b s v}(n)=L_{o b s v}(n-1)+\left(f_{2}(n) v_{2}(n)+\alpha(n-\right.$ 1) $\left.v_{2}(n-1)^{2}\right)+b \dot{v}_{2}^{2}(n)$

5)

$$
\alpha(n)= \begin{cases}-L_{o b s v} / v_{2}(n)^{2} & \text { if } L_{o b s v}<0 \\ 0 & \text { if } L_{o b s v} \geq 0\end{cases}
$$

6) Output $\Longrightarrow f_{1}(n)=f_{2}(n)+\alpha(n) v_{2}(n)$

\section{Evaluation}

To evaluate the stabilizing performance of the proposed LOLC approach in wider range of impedance, the numerical simulation has been carried with a basic haptic interface consisting of human operator (HO), haptic interface (HI), and a virtual environment (VE). The VE consist of a firstorder mass spring damper model that is updated at $100 \mathrm{~Hz}$. In addition, the haptic probe was initially locate at $-0.004 \mathrm{~m}$ and the VE was located at $0.0 \mathrm{~m}$. The $\mathrm{HO}$ and $\mathrm{HI}$ models were modelled with following parameters.

For the human operator $m_{H O}=0.1 \mathrm{~kg}, b_{H O}=0.2 \mathrm{~N} . \mathrm{s} / \mathrm{m}, k_{H O}=50.0 \mathrm{~N} / \mathrm{m}$

For haptic interface $m_{H I}=0.2 \mathrm{~kg}, b_{H I}=0.001 \mathrm{~N} . \mathrm{s} / \mathrm{m}$

We tuned the inertia and damping value down within a reasonable range to reduce the energy dissipation by the haptic device. However, it is difficult to set human operator parameters since it is identical to operating environments, and the control approach should assure the stability of the system despite of human parameters. In this evaluation, the model is tested for high stiffness values. Therefore, human operator model is designed with a low damping value to avoid dissipating the energy generated by the system.

First, to check the performance of the proposed approach in face of high stiffness virtual environments, a relatively high stiffness value $(\mathrm{k}=8 \mathrm{kN} / \mathrm{m})$ and low virtual damping $(b=2 \mathrm{Nm} / \mathrm{s}$ ) is introduced to the VE. The position response 


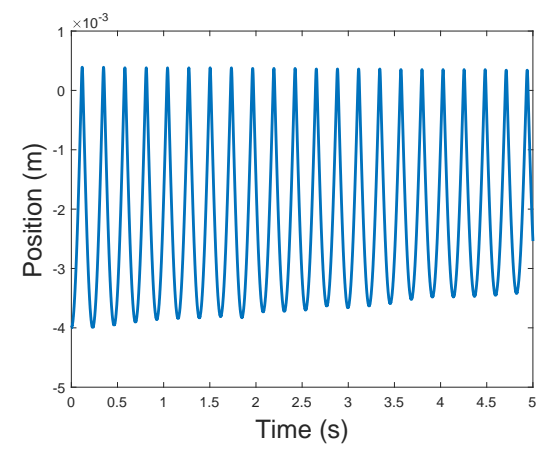

Fig. 6: Position response with $8 \mathrm{kN} / \mathrm{m}$ stiffness VE without the LC

shows a high instability without the LC, generating constant oscillations in position response as show in the Fig. 6. Next, the proposed LOLC approach is introduced to the unstable system to stabilize the position response. As shown in Fig. 7 (a), the position response of the VE, get stabilize with less oscillations. Fig. 7 (b), shows the un-revised force which is the output of the VE. It has generated a peak around $3 \mathrm{~N}$ at the beginning and the force is reduced for $t>0$ letting the system stable. That is because, the LC has generated force with the same magnitude, but in opposite direction as shown in the Fig. 7(c). Hence, the force which has been leading to instability is suppressed by the LC force. However, in the VE $m$. In the Fig. 7(d),it shows that the energy of the system has reduced for $t>0$, due to the dissipating action of the LC. Hence, the energy of the system reduced from $2 \times 10^{-4}$ $\mathrm{Nm}$ to zero energy level, which is the energy threshold in the proposed LOLC approach. In addition, the energy of the system keep remaining in zero-level with out any oscillations which guarantee a stable response.

Finally, LOLC approach is compared with the POPC approach [2] in a VE with stiffness of $50 \mathrm{kN} / \mathrm{m}$ and damping of $20 \mathrm{Nm} / \mathrm{s}$ for analysing the response for high stiffness environments. Both the approaches were able to stabilize the system, while POPC approach stabilized the system in less time and show low amplitude in the oscillations before stabilize, compared to LOLC approach (Fig. 8).

Due to the less conservativeness of the LOLC approach, the system has dissipated less energy within one sampling interval which causes for the longer settling time of the response. As shown in the magnified section of Fig. 8, after stabilizing with the LOLC, the position response has remained smoother as the POPC approach has shown some jittering in the position response after stabilized due to its conservative criteria which activate the PC.

As shown in the Fig. 9, the POPC has shown a impulsive behaviour in the energy flow while the LOLC has a smooth behaviour in energy flow. The magnified section in Fig. 9 shows, the smoothness of energy flow in the LOLC approach and less energy dissipation. This smooth behaviour results in having small amount of energy remaining in the system even after stabilizing, as shown by the small peaks in the Fig. 9. However, this remaining energy does not affect the

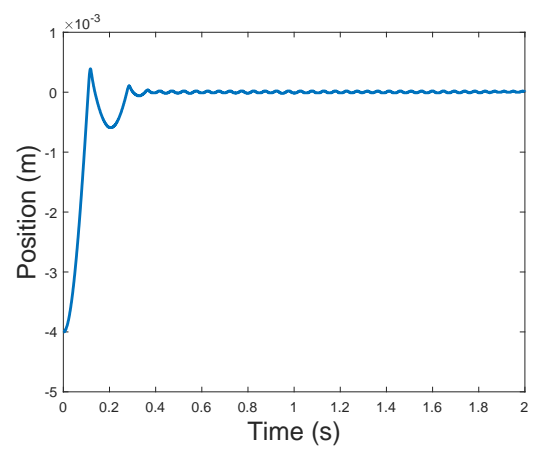

(a)

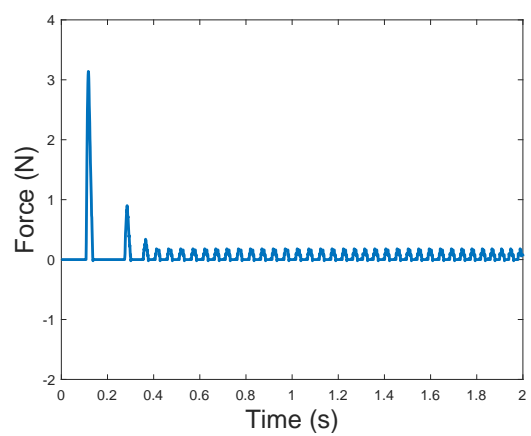

(b)

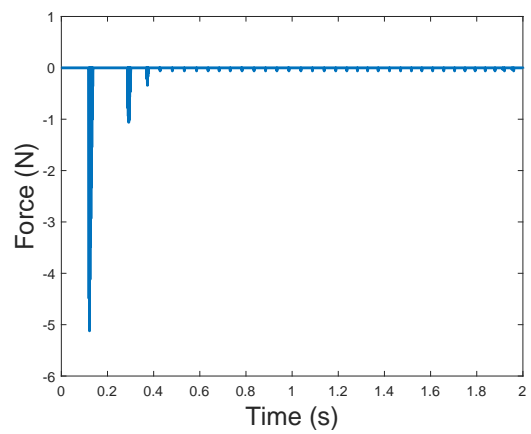

(c)

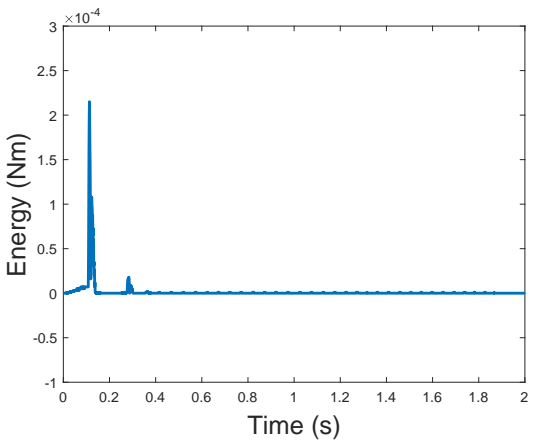

(d)

Fig. 7: System performance with stiffness $8 \mathrm{kN} / \mathrm{m}$ and damping $2 \mathrm{Nm} / \mathrm{s}$ with the LOLC ; (a) Position response (b) Un-revised force (c) LC generated force (d) Energy 


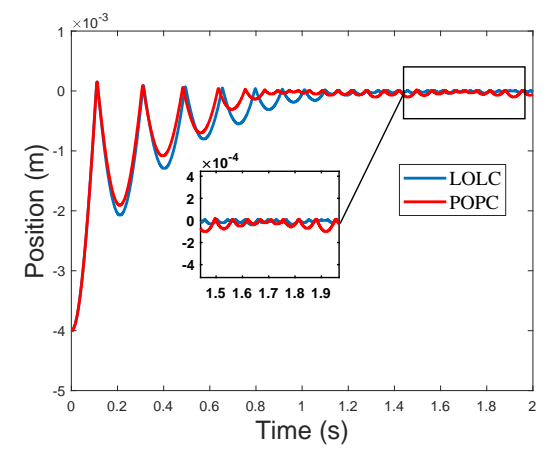

Fig. 8: Position response of the POPC and the LOLC at stiffness $=50$ $\mathrm{kN}$ and damping $=20 \mathrm{Nm} / \mathrm{s}$

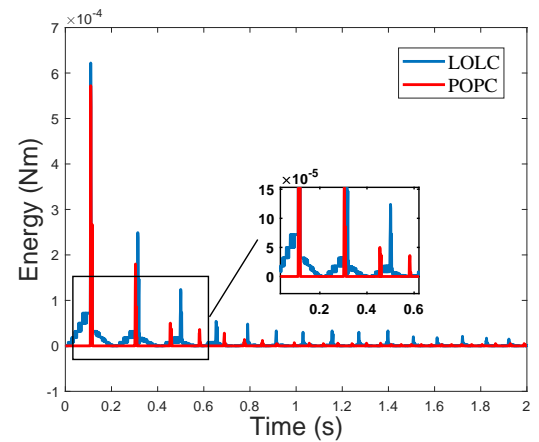

Fig. 9: Energy of the observer at stiffness $=50 \mathrm{kN} / \mathrm{m}$ and damping $=20$ $\mathrm{Nm} / \mathrm{s}$ in the POPC and LOLC approaches

instability of the system since it is controlled by the LC force continuously. Hence, with the LOLC approach, the system does not dissipates whole energy and continues to have less energy in the system close to observer threshold. Therefore, the position response of the system (Fig. 8) has no oscillation and continues to have a stable response for all $t>0$.

\section{CONCLUSion AND Future Work}

To reduced the conservatism of passivity-based approaches, this paper proposed a novel Lyapunov observer and controller to achieve a stable haptic interaction. The proposed approach is not limited for haptic interfaces, but also could be used as a general control architecture with a less-conservative criteria. By using the Lyapunov boundedness theorem, a Lyapunov Observer (LO) was proposed to check the satisfaction of the Lyapunov boundedness theorem at each and every sampling time. If the satisfaction was not met, a Lyapunov controller (LC) was proposed to correct the deviation from the Lyapunov boundedness criteria using a virtual damper. This paper also discussed implementation of LO and LC for a one-port network. A designed VE consisting of mass, spring and damper is used to show the numerical evaluation of the LOLC approach. These numerical studies show that LOLC approach is less conservative than the POPC approach, although both approaches are well respond to high stiffness environments.

In this paper, we demonstrated the effectiveness of the proposed approach numerically. Therefore, as the next step, we are going to evaluate the proposed approach experimentally on a haptic device. Moreover, one of the main advantages of the proposed approach is that it is not limited to stabilize only the haptic devices, and the main idea could be applied to any controlled system. As a future work, we are considering the LOLC for stabilizing a test rig which has been designed to test prosthetic legs.

\section{ACKNOWLEDGMENT}

This research was supported by the UK Engineering and Physical Sciences Research Council (EPSRC) under grant reference EP/P022588/1, and the grant "Development of core teleoperation technologies for maintaining and repairing tasks in nuclear power plants" funded by the Ministry of Trade, Industry and Energy of S. Korea, and Industrial Strategic Technology Development Program (10052967) funded by the Ministry of Trade, Industry and Energy of S. Korea. The authors also acknowledge the support of Professor Chris Melhuish from the Bristol Robotics Laboratory, University of the West of England.

\section{REFERENCES}

[1] T. H. Do and J.-H. Ryu, "Memory based passivation method for stable haptic interaction," in World Haptics Conference (WHC), 2011 IEEE, pp. 409-414, IEEE, 2011.

[2] B. Hannaford and J.-H. Ryu, "Time-domain passivity control of haptic interfaces," IEEE Transactions on Robotics and Automation, vol. 18, no. 1, pp. 1-10, 2002.

[3] J.-P. Kim and J. Ryu, "Energy bounding algorithm based on passivity theorem for stable haptic interaction control," in Haptic Interfaces for Virtual Environment and Teleoperator Systems, 2004. HAPTICS'04. Proceedings. 12th International Symposium on, pp. 351-357, IEEE, 2004

[4] J.-H. Ryu and M.-Y. Yoon, "Memory-based passivation approach for stable haptic interaction," IEEE/ASME Transactions on Mechatronics, vol. 19 , no. 4 , pp. 1424-1435, 2014.

[5] F. Llewellyn, "Some fundamental properties of transmission systems," Proceedings of the IRE, vol. 40, no. 3, pp. 271-283, 1952.

[6] A. Jafari, M. Nabeel, and J. H. Ryu, "The input-to-state stable (iss) approach for stabilizing haptic interaction with virtual environments," IEEE Transactions on Robotics, vol. 33, pp. 948-963, Aug 2017.

[7] E. D. Sontag and Y. Wang, "On characterizations of the input-to-state stability property," Systems \& Control Letters, vol. 24, no. 5, pp. 351$359,1995$.

[8] E. D. Sontag, Input to State Stability: Basic Concepts and Results, pp. 163-220. Berlin, Heidelberg: Springer Berlin Heidelberg, 2008.

[9] A. V. der Schaft, L2-Gain and Passivity Techniques in Nonlinear Control. New York,NY,USA: Springer, 2012.

[10] H. K. Khalil, "Noninear systems," Prentice-Hall, New Jersey, vol. 2 , no. 5, pp. 5-1, 1996. 